\title{
BMJ Open Safety and efficacy of tranexamic acid in paediatric cardiac surgery: study protocol for a double-blind randomised controlled trial
}

\author{
Yu Zhang, ${ }^{1}$ Yuan Jia, ${ }^{1}$ Jia Shi, ${ }^{1}$ Su Yuan, ${ }^{1}$ Rong Wang, ${ }^{1}$ Zhe Zhang, ${ }^{1}$ Xu Wang, ${ }^{2}$ \\ Jinping Liu, ${ }^{3}$ Jun Ran, ${ }^{4}$ Yuchen Zhao, ${ }^{4}$ Zhongdong Hua, ${ }^{4}$ Jun Yan, ${ }^{4}$ Shoujun Li, ${ }^{4}$ \\ Zhe Zheng, ${ }^{4}$ Shengshou Hu, ${ }^{4}$ Yang Wang, ${ }^{5}$ Fuxia Yan (D) ${ }^{1}$
}

To cite: Zhang Y, Jia Y, Shi J, et al. Safety and efficacy of tranexamic acid in paediatric cardiac surgery: study protoco for a double-blind randomised controlled trial. BMJ Open 2019;9:e032642. doi:10.1136/ bmjopen-2019-032642

- Prepublication history for this paper is available online To view these files, please visit the journal online (http://dx.doi org/10.1136/bmjopen-2019032642).

Received 30 June 2019 Revised 30 0ctober 2019 Accepted 30 0ctober 2019

\section{Check for updates}

(c) Author(s) (or their employer(s)) 2019. Re-use permitted under CC BY-NC. No commercial re-use. See rights and permissions. Published by BMJ.

For numbered affiliations see end of article.

\section{Correspondence to} Dr Fuxia Yan; yanfuxiafw@sina.com

Professor Yang Wang; wangyfuwai@sohu.com

\section{ABSTRACT}

Introduction An initial retrospective study suggested that tranexamic acid (TXA) administration increased the incidence of seizures in paediatric patients undergoing cardiac surgery. However, the efficacy of TXA in paediatric cardiac surgery remains unclear owing to the small sample sizes of the studies. Therefore, this study will investigate the efficacy and safety of TXA in paediatric patients undergoing cardiac surgery. We hypothesised that TXA may increase the incidence of postoperative seizures with no effect on postoperative allogeneic transfusion in paediatric patients undergoing cardiac surgery. The pragmatic study will provide important implications for paediatric cardiac surgery.

Methods and analysis This will be a single-centre prospective, double-blind randomised controlled trial. The plan is to enrol in the study 2090 paediatric patients aged 31 days to 7 years who will be undergoing cardiac surgery with cardiopulmonary bypass (CPB). All eligible participants will be randomly assigned to either the TXA or placebo group by using a Web-based randomisation service in a 1:1 ratio. The primary safety end point will be postoperative seizures until hospital discharge, and the primary efficacy end point will be the volume of allogeneic red blood cell transfusion after termination of CPB. All patients will be followed up for 1 year postdischarge. All data will be analysed in accordance with the intention-totreat principle.

Ethics and dissemination This study was approved by the institutional review board of Fuwai Hospital, Chinese Academy of Medical Sciences and Peking Union Medical College (No 20191195). Written informed consent will be obtained from the parents/legal guardian of each patient because all participants will be $<18$ years of age. The results of the trial will be published in an international peer-reviewed journal.

Trial registration number Chinese Clinical Trial Register (ChiCTR1900024131).

\section{INTRODUCTION}

Paediatric cardiac surgery increases the risk of excessive blood loss and subsequent allogeneic transfusion due to the abnormal coagulation in patients with congenital heart
Strengths and limitations of this study

- This will be a randomised controlled trial aimed at evaluating the efficacy and safety of tranexamic acid (TXA) in paediatric patients undergoing cardiac surgery, which will provide important implications.

- More than 2090 participants will be recruited to obtain results with high statistical power.

- The TXA dosage that will be used in this study is appropriate according to a pharmacokinetic study.

- This will be a single-centre trial, which can be a limitation of this study.

- This study will exclude some high-risk patients, such as neonates and children undergoing total cavopulmonary connection, under the consideration of heterogeneity.

disease and the activation of platelets, coagulation and fibrinolysis caused by hemodilution during cardiopulmonary bypass (CPB). ${ }^{1}$ Moreover, allogeneic transfusion is associated with increases in the incidence of postoperative pulmonary complications, mechanical ventilation duration, hospital length of stay and total hospitalisation cost in paediatric patients undergoing cardiac surgery. ${ }^{2}$ Antifibrinolytic therapy, including tranexamic acid (TXA), aminocaproic acid and aprotinin, is currently an important measure for blood protection in paediatric cardiac surgery. However, a series of studies suggested that aprotinin administration increases the incidence of renal insufficiency and mortality, and the use of the drug was therefore suspended in 2008. TXA has since become the primary antifibrinolytic drug in paediatric cardiac surgery. ${ }^{3}$

Some studies have shown that TXA reduces both postoperative bleeding and allogeneic transfusion in paediatric cardiac surgery. ${ }^{45}$ However, as the current guideline indicates, 
clinical studies on the use of TXA in paediatric cardiac surgery have been limited by their small sample sizes; thus, TXA administration is not explicitly recommended in paediatric cardiac surgery. ${ }^{6} \mathrm{~A}$ large retrospective cohort study $^{7}$ and a randomised trial ${ }^{8}$ showed that TXA administration was associated with a reduction in the postoperative bleeding volume but not the allogeneic transfusion requirement in paediatric patients undergoing cardiac surgery.

Considering the clinical experience with aprotinin, more attention should be paid to the adverse effects of TXA. Pasquali $e t a t^{\theta}$ suggested that use of TXA was associated with significantly lower mortality than that of aprotinin in paediatric cardiac surgery. Unfortunately, recent clinical trials and meta-analyses have suggested a dosedependent association between TXA administration and the risk of seizures in adult patients undergoing cardiac surgery. ${ }^{10-12}$ Moreover, recent studies have suggested that a TXA dose of $>100 \mathrm{mg} / \mathrm{kg}$ confers a twofold increased risk of seizures when compared with lower doses $(30-50 \mathrm{mg} / \mathrm{kg})$. Retrospective studies have revealed that the use of TXA is associated with a significantly increased risk of seizures in paediatric cardiac surgery. ${ }^{13-15}$ Maeda et $a l^{15}$ found a $0.2 \%$ incidence of seizures in the nonTXA group and a $1.6 \%$ incidence of seizures in the TXA group among paediatric patients undergoing cardiac surgery $(p<0.001)$. However, the adverse effects to TXA in paediatric cardiac surgery have only been reported in retrospective studies; no prospective studies have been designed or powered to evaluate the incidence of adverse events. Moreover, seizures after cardiac surgery may increase the operative mortality and interfere with the neurodevelopment and quality of life of children. ${ }^{16} 17$ Therefore, safety evaluations of the use of TXA in paediatric cardiac surgery are important.

On the basis of our preliminary observations in clinical practice, we hypothesised that TXA may increase the incidence of postoperative seizures while having no effect on postoperative allogeneic transfusion in paediatric patients undergoing cardiac surgery. Therefore, although removing TXA from routine treatment may have no effect on perioperative blood protection, this may greatly reduce the risk and cost of surgery and improve the longterm neurodevelopment and quality of life of paediatric patients undergoing cardiac surgery.

To verify our hypothesis, we designed a large randomised controlled trial (RCT) to investigate the efficacy and safety of TXA in paediatric patients undergoing cardiac surgery.

\section{METHODS AND ANALYSIS \\ Study objective}

The aim of our study will be to test the hypothesis that TXA, compared with placebo, increases the incidence of postoperative seizures and has no effect on postoperative allogeneic transfusion in paediatric patients undergoing cardiac surgery.
Recruitment of potential subjects willing to participate

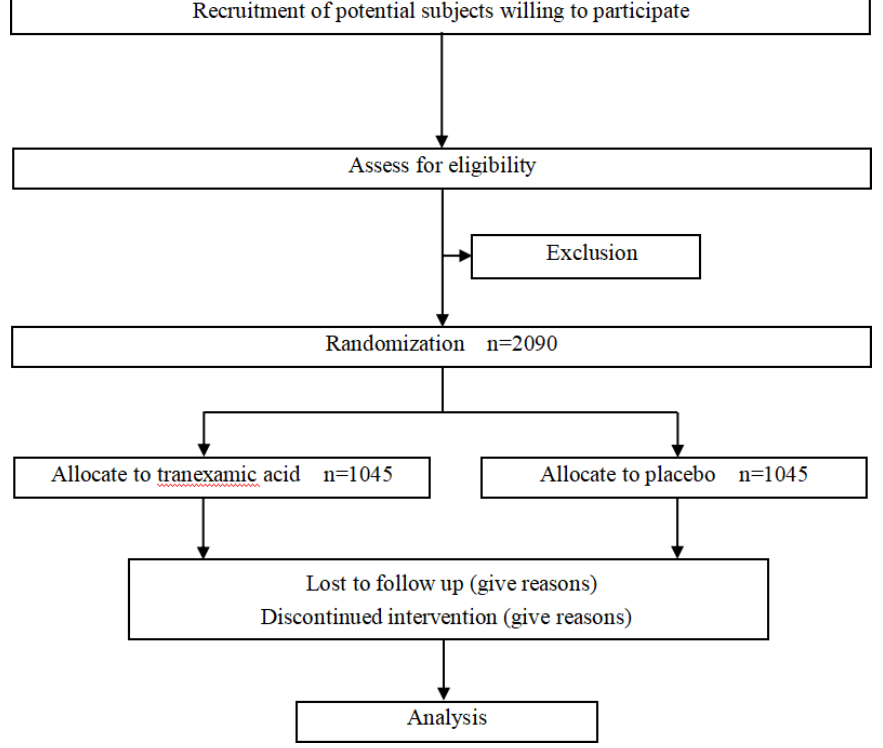

Figure 1 Flowchart of the study.

\section{Study design}

The study will be a single-centre, parallel-group, doubleblind RCT. The study protocol adheres to the Standard Protocol Items: Recommendations for Interventional Trials guideline. Patients will first be screened for eligibility and willingness to participate in the trial. Informed consent will then be collected from all the participants. Written informed consent will be obtained from the parents/legal guardian of each patient because all participants will be $<18$ years of age. All eligible participants will be randomly assigned to either the TXA or placebo group in a 1:1 ratio in a blinded fashion. Randomisation will be performed by using a Web-based randomisation service in Fuwai Hospital. The patients will be closely monitored for the occurrence of postoperative seizures. The times of events will also be assessed. The study flowchart is shown in figure 1.

\section{Study population}

We plan to enrol 2090 paediatric patients aged 31 days to 7 years who will be undergoing cardiac surgery with CPB at Fuwai Hospital in Beijing, China. The included patients will be undergoing surgical repair of an atrial or ventricular septal defect, atrioventricular septal defect, or endocardial cushion defect, or a complete repair for tetralogy of Fallot, double-outlet right ventricle, transposition of the great arteries, pulmonary atresia, anomalous pulmonary venous connection, anomalous origin of the left coronary artery from the pulmonary artery, and similar conditions. The patients will be included in the study after their parents/guardian provide written informed consent. The exclusion criteria will be total cavopulmonary connection with $\mathrm{CPB}$, thrombocytopenia or any other known history of a bleeding disorder, thromboembolic disease (history of postoperative or spontaneous pulmonary embolism, spontaneous arterial thrombosis or familial hypercoagulability), previous convulsion or seizure, intellectual or 
legal disabilities, severe renal impairment (serum creatinine level of $>3.3 \mathrm{mg} / \mathrm{dL}$ ), allergy or contraindication to TXA injection or its components, and current enrolment in another perioperative interventional study.

\section{Randomisation and blinding}

Randomisation will be performed using a Web-based randomisation service in Fuwai Hospital. The allocation sequence will be computer-generated, and the randomisation list will not be known to the investigators. All eligible participants will be randomly assigned to either the TXA or placebo group in a 1:1 ratio. The participants, medical staff and investigators will be unaware of the treatment allocation. The drugs will be prepared by nurse anaesthetists, labelled with numbers and then injected by the anesthesiologist. Data will be collected by trained observers who will not participate in patient care and will be blinded to the patient allocation.

\section{Interventions}

The participants will be randomised to receive injections using identical syringes labelled with the randomisation number. The syringes will contain a transparent solution (either TXA or saline solution). In infants aged 31 days to 12 months, the medication will be intravenously administered with a pump in a $30 \mathrm{mg} / \mathrm{kg}$ bolus over a $15 \mathrm{~min}$ period after induction, followed by maintenance at $10 \mathrm{mg} /(\mathrm{kg} \cdot \mathrm{h})$ throughout the surgery. In children aged 12 months to 7 years, the medication will be intravenously administered with a pump in a $10 \mathrm{mg} / \mathrm{kg}$ bolus over a $15 \mathrm{~min}$ period after induction, followed by maintenance at $10 \mathrm{mg} /(\mathrm{kg} \cdot \mathrm{h})$ throughout the surgery.

All other perioperative clinical care will be performed in accordance with the standard practice in our centre because this trial is designed to represent real-world clinical practice. Heparinisation for CPB will be based on a bolus dose of $400 \mathrm{U} / \mathrm{kg}$ and maintenance of an activated clotting time of $>450 \mathrm{~s}$ during $\mathrm{CPB}$, with additional heparin as required. Heparin reversal at CPB completion will be achieved with protamine administration at $4 \mathrm{mg}$ / $\mathrm{kg}$, monitored on the basis of the activated clotting time $(<140$ s). Other antifibrinolytic therapies such as human fibrinogen, human prothrombin and recombinant VIIa cannot be used before or during CPB; however, these can be used if clinically significant bleeding is present after protamine administration. The study will be guided by a transfusion protocol. The threshold for red blood cell transfusion will be a haemoglobin concentration of $<100 \mathrm{~g} / \mathrm{L}$ in acyanotic patients and $<110 \mathrm{~g} / \mathrm{L}$ in cyanotic patients after CPB termination. The indication for fresh frozen plasma administration will be an activated partial thromboplastin time of $>48 \mathrm{~s}$, prothrombin time of $>21 \mathrm{~s}$ or bleeding volume of $>4 \mathrm{~mL} /(\mathrm{kg} \cdot \mathrm{h})$ at any time. Concentrated platelets will be administered when the platelet count decreases to $<50 \times 10^{9} / \mathrm{L}$. In addition, the participants will be followed up by means of an in-hospital review, telephone or remote video interview via a smartphone and social media at 30 days and 1 year after surgery. Their medical records will be reviewed to determine whether they have experienced any adverse events or death.

\section{End points and definitions}

The primary end points will be safety and efficacy. The primary safety end point will be postoperative seizures until hospital discharge, and the primary efficacy end point will be the volume of allogeneic red blood cell transfusion after CPB termination. The secondary safety end points will be stroke, myocardial infarction, pulmonary embolism, deep venous thrombosis, acute kidney injury (AKI) and all-cause mortality until hospital discharge or 30 days and 1 year after surgery; seizures at 30 days and 1 year after surgery. The secondary efficacy end points will be exposure to allogeneic red blood cell transfusion after CPB termination, volume of and exposure to fresh frozen plasma and platelet transfusions after CPB termination. Additionally, other study variables will be postoperative bleeding (6-hour postoperative bleeding to be measured as the accumulated volume of pericardial and mediastinal fluid collected via a drainage tube during the first 6 hours after surgery and total postoperative bleeding to be measured before removal of the tubes); the rate of reoperation for massive bleeding with a drainage rate of $>10 \%$ of the total blood volume per hour for up to 2 hours or until the occurrence of cardiac tamponade; mechanical ventilation duration; intensive care unit stay; hospital length of stay; and total hospitalisation cost.

Seizure will be defined as 'a transient occurrence of signs and/or symptoms due to abnormal excessive or synchronous neuronal activity in the brain'. Seizures will be usually clinically manifested as tonic, clonic or myoclonic activity of the limb or trunk, or generalised all over the body with or without loss of awareness. All the patients with seizures will undergo serial electroencephalography, which may show epileptiform discharges, or slow waves or normal findings.

Stroke will be diagnosed as a new neurological deficit (paralysis, weakness or speech difficulty) that lasts for $>24$ hours or that leads to early death and will be confirmed on the basis of the presence of cerebral infarction or haemorrhage on CT or MRI.

Myocardial infarction will be defined as the development of pathologic $Q$ waves on two or more adjacent leads on an ECG, changes indicative of ischemia (ST-segment elevation or depression) on an ECG, and/ or a change in the serum level of creatine kinase-muscle/ brain of $>75 \mu \mathrm{g} / \mathrm{L}$.

Pulmonary embolism will be confirmed on the basis of clinical symptoms and pulmonary angiography or ultrasonography findings.

Deep venous thrombosis will be confirmed on the basis of clinical symptoms and venous Doppler ultrasonography findings.

AKI will be defined in accordance with the Acute Kidney Injury Network criteria. ${ }^{18}$ The ratio of the peak serum creatinine level to the preoperative serum creatinine 
level on each postoperative day will be used to define the change in renal function. The patient will be considered to have mild AKI if the highest ratio attained is 1.5 to 2.0, moderate AKI if the highest ratio is 2.0 to 3.0, and severe AKI if the serum creatinine level is $\geq 4.0 \mathrm{mg} / \mathrm{dL}$, the peak ratio is $>3.0$, or renal replacement therapy is required.

\section{Sample size estimation}

The study hypothesis is that TXA increases the incidence of postoperative seizures while having no effect on postoperative allogeneic transfusion in paediatric patients undergoing cardiac surgery. The sample size calculation will be mainly based on the incidence of postoperative seizures and the volume of red blood cell transfusion.

For the primary safety end point, we determined that a sample size estimate of 1902 randomised subjects (951 for each group) has $90 \%$ power to detect an increase in the incidence of postoperative seizures from $0.2 \%$ in the non-TXA group to $1.6 \%$ in the TXA group, on the basis of the most recent large retrospective study, ${ }^{15}$ by means of a single-sided $\alpha=0.025$.

For the primary efficacy end point, we determined that a sample size estimate of 264 randomised subjects (132 for each group) has $90 \%$ power to detect a noninferiority margin for a $20 \mathrm{~mL}$ difference in the volume of red blood cell transfusion with a SD of $50 \mathrm{~mL}$, on the basis of a study by Giordano $e t a l,{ }^{19}$ by means of a single-sided $\alpha=0.025$.

To conduct an interim analysis, the sample size in each group was determined to be 957 for the adjusted significance level (from 0.025 to 0.0245 in accordance with the alpha spending function by the Lan-DeMets method).

Finally, assuming crossovers, protocol violation and a loss-to-follow-up rate of $8 \%$, we calculated that we would need a sample size of 2090 participants (1:1 ratio) for the clinical trial.

\section{Statistical analysis}

A statistician who has extensive experience in designing, conducting and analysing clinical trials; will not be involved in the patient management; and will be blinded to the assigned intervention will be responsible for the statistical analysis.

Data will be stored electronically via a Web-based case report form and analysed using SAS V.9.2. All data will be analysed in accordance with the intention-to-treat principle, beginning immediately after randomisation.

Normally distributed continuous variables will be shown as mean and SD, and compared using the Student's t-test. Non-normally distributed continuous variables will be shown as median and IQR, and compared using the Wilcoxon-Mann-Whitney test. Categorical variables will be presented as frequency and percentage, and compared using the $\chi^{2}$ test or Fisher's exact test. If the baseline characteristics show significant differences between the two groups, regression analysis will be used. A general linear regression model will be used to analyse the bleeding volume and allogeneic transfusion volume. A logistic regression model will be used to analyse allogeneic transfusion exposure and the outcome events. If the outcome event proves to be rare, a Poisson regression model will be used. A Cox regression model will be used to analyse the follow-up morbidity and mortality rates. The number of covariates in the regression model will be decided on the basis of the number of outcome events.

An independent data and safety monitoring board (DSMB) will perform one interim analysis after recruitment of $50 \%$ of the 2090 participants. The interim analysis will be adjusted according to the Lan-DeMets alpha spending function for the TXA main effects. For the interim analysis, a single-sided $\mathrm{p}$ value $<0.0015$ will be taken as the stopping rule.

\section{Data and safety monitoring board}

The DSMB will consist of a cardiologist (chair), cardiac surgeon, independent statistician, cardiac anesthesiologist with an interest in medical ethics and law, and clinical pharmacologist. The independent DSMB will perform one interim analysis after recruitment of $50 \%$ of the 2090 participants, and make recommendations to continue, modify or terminate the study according to the stopping rules and consideration of other evidence relevant to the DSMB.

\section{Study status}

The trial will start patient recruitment in December 2019, and the recruitment will continue for 2 years, until December 2021. The 1year follow-up programme is planned to be continued until December 2022.

\section{Patient and public involvement}

No patient or public entity will be involved in the present study. On the completion of this trial, a journal article manuscript will be prepared to present the trial results.

\section{DISCUSSION}

To the best of our knowledge, this is currently the first large RCT to evaluate the efficacy and safety of TXA in paediatric cardiac surgery, which will provide important implications for paediatric cardiac surgery. If our hypothesis is proven correct, removing TXA from routine treatment may have no effect on perioperative blood protection but may greatly reduce the risk and cost of surgery and improve the long-term neurodevelopment and quality of life of paediatric patients undergoing cardiac surgery.

Owing to the increased risk of renal failure and mortality reported in adults undergoing cardiac surgery, aprotinin administration should not be considered as a safe option for children. Since withdrawal of aprotinin from the market in 2008, TXA has been widely used as an antifibrinolytic agent. Unfortunately, evidence is limited regarding the safety of TXA in paediatric cardiac surgery. Pasquali $e t a \ell^{9}$ performed a large Society of Thoracic Surgeons National Database study of $>20000$ paediatric patients undergoing cardiac surgery and found that TXA (vs aprotinin) was associated with significantly reduced mortality/bleeding 
requiring surgical intervention overall $(\mathrm{OR}, 0.47 ; 95 \%$ CI 0.30 to 0.74 ) and in neonates (OR, $0.30 ; 95 \%$ CI 0.15 to 0.58 ). Therefore, TXA was shown to be superior to aprotinin in terms of transfusion with less morbidity and mortality in paediatric cardiac surgery. However, several retrospective cohort studies revealed adverse events related to TXA administration during paediatric cardiac surgery. ${ }^{13-15}$ Martin et al ${ }^{1314}$ found $9.6 \%, 1.8 \%, 3.5 \%$ and $2.6 \%$ incidence rates of renal injury, renal failure, seizure and other neurological events, respectively. According to their results, $>500$ patients per group are needed to evaluate the adverse effects of TXA administration in paediatric cardiac surgery. In fact, according to the findings reported by Maeda et al, ${ }^{15}$ our planned study of the effects of TXA in a very large number of participants $(n=2090)$ will be powerful enough to explain the safety of TXA in paediatric cardiac surgery.

At present, the dosage of TXA recommended for use during paediatric cardiac surgery significantly vary. The loading dose of TXA ranges from 10 to $100 \mathrm{mg} / \mathrm{kg}$, and the maintenance dose ranges from 1 to $15 \mathrm{mg} /(\mathrm{kg} \cdot \mathrm{h}){ }^{20}$ The dosage regimens that will be used in our study will be based on pharmacokinetic principles. Multiple in vitro studies have focused on determining the TXA concentration needed to prevent fibrinolysis, inhibit platelet activation or increase thrombin generation. Paediatric cardiac TXA pharmacokinetic studies have shown that a lower plasma concentration of TXA $(\sim 10-20 \mu \mathrm{g} / \mathrm{mL})$ is thought to inhibit $\sim 80 \%$ of fibrinolysis and that a high plasmatic concentration of TXA $(\sim 100 \mu \mathrm{g} / \mathrm{mL})$ should completely inhibit fibrinolysis. ${ }^{21} 22$ On the basis of the plasma concentration of TXA obtained from these in vitro models, a low dosage maintaining a low plasmatic concentration of TXA may not achieve the effect of clinical blood protection, while a high dosage may cause adverse events after paediatric cardiac surgery. In a recent paediatric cardiac TXA pharmacokinetic study, Wesley et $a l^{23}$ described three different dose regimens based on targeted plasmatic concentrations of 20,60 and $150 \mu \mathrm{g} / \mathrm{mL}$. To maintain a plasma concentration of $60 \mu \mathrm{g} / \mathrm{mL}$, the authors suggested a loading doses of 50,26 and $13 \mathrm{mg} / \mathrm{kg}$ followed by a continuous infusion of 7,6 and $5.5 \mathrm{mg} /(\mathrm{kg} \cdot \mathrm{h})$ in neonates and infants aged $<2,2-12$ and $>12$ months and $\leq 20 \mathrm{~kg}$ in body weight, respectively. An additional priming volume of $60 \mu \mathrm{g} / \mathrm{mL}$ was also suggested. Therefore, on the basis of the existing literature, the present study indicates that a TXA loading dose of $30 \mathrm{mg} / \mathrm{kg}$ followed by a continuous infusion of $10 \mathrm{mg} /(\mathrm{kg} \cdot \mathrm{h})$ can be used in infants $<1$ year of age, while a loading dose of $10 \mathrm{mg} / \mathrm{kg}$ followed by a continuous infusion of $10 \mathrm{mg} /(\mathrm{kg} \cdot \mathrm{h})$ can be used in older patients; this is recommended by Faraoni $e t$ $a l^{24}$ as an expert opinion.

\section{Limitations}

The single-centre nature of this study may limit its generalisability; thus, a multicentre study with a large sample size will be needed in the future. Although the age range of the patients is broad, as is the number of procedures that will be included, this RCT will be a pragmatic study. If the baseline characteristics show significant differences between the two groups, multivariate adjustment will be used to reduce overt bias. In addition, the present study will exclude some high-risk patients such as neonates and children undergoing total cavopulmonary connection. This may limit the generalisability of the research. Neonates aged $<1$ month have an immature coagulation system and fibrinolysis disorders; thus, the requirement for postoperative allogeneic transfusion in this age group is much higher than that in other age groups. ${ }^{25}$ Paediatric patients undergoing total cavopulmonary connection in our centre are older than those in developed countries because of the limited economic conditions in developing countries. Moreover, children undergoing total cavopulmonary connection in our centre have poorer pulmonary artery development and higher pulmonary artery pressure, which increase the postoperative serosanguineous and allogeneic transfusion requirement, than those in American and European centres. Therefore, they will be excluded under the consideration of heterogeneity. Nevertheless, we believe that the clinical evaluations outlined in this protocol will have important implications for paediatric cardiac surgery. Furthermore, the effects of TXA administration in neonates undergoing cardiac surgery and paediatric patients undergoing total cavopulmonary connection need to be further studied.

\section{CONCLUSIONS}

The pragmatic study will be the first adequately powered RCT to evaluate the efficacy and safety of TXA in paediatric patients undergoing cardiac surgery, which will provide important implications for paediatric cardiac surgery. If the predicted effects are proven, removing TXA from routine treatment may have no effect on perioperative blood protection, but may greatly reduce the risk and cost of surgery and improve the long-term neurodevelopment and quality of life of paediatric patients undergoing cardiac surgery.

\section{Author affiliations}

${ }^{1}$ Anaesthesiology, Fuwai Hospital, National Centre for Cardiovascular Diseases, Chinese Academy of Medical Sciences and Peking Union Medical College, Beijing, China

${ }^{2}$ Paediatric Intensive Care Unit, Fuwai Hospital, National Centre for Cardiovascular Diseases, Chinese Academy of Medical Sciences and Peking Union Medical College, Beijing, China

${ }^{3}$ Cardiopulmonary Bypass, Fuwai Hospital, National Centre for Cardiovascular Diseases, Chinese Academy of Medical Sciences and Peking Union Medical College, Beijing, China

${ }^{4}$ Cardiovascular Surgery, Fuwai Hospital, National Centre for Cardiovascular Diseases, Chinese Academy of Medical Sciences and Peking Union Medical College, Beijing, China

${ }^{5}$ Medical Research and Biometrics Centre, National Centre for Cardiovascular Diseases, Chinese Academy of Medical Sciences and Peking Union Medical College, Beijing, China

Contributors $Y Z, Y W$ and $F Y$ conceived the study and initiated the study design. YZ, YJ, JS, SY, RW, ZZhang, XW, JL, JR, Y-CZ, ZH and JY will be involved in study implementation. YW provided statistical expertise in clinical trial design and will 
conduct the statistical analysis. SL, ZZheng, SH and FY will provide expertise with data interpretation. All authors participated, read and approved the final manuscript.

Funding The study is supported by the Fundamental Research Funds for the Central Universities of Peking Union Medical College (No 3332018064).

Competing interests None declared.

Patient consent for publication Parental/guardian consent obtained.

Ethics approval The study was approved by the institutional review board of Fuwai Hospital, Chinese Academy of Medical Sciences and Peking Union Medical College (No 20191195).

Provenance and peer review Not commissioned; externally peer reviewed.

Open access This is an open access article distributed in accordance with the Creative Commons Attribution Non Commercial (CC BY-NC 4.0) license, which permits others to distribute, remix, adapt, build upon this work non-commercially, and license their derivative works on different terms, provided the original work is properly cited, appropriate credit is given, any changes made indicated, and the use is non-commercial. See: http://creativecommons.org/licenses/by-nc/4.0/.

ORCID iD

Fuxia Yan http://orcid.org/0000-0001-5604-1328

\section{REFERENCES}

1 Siemens K, Sangaran DP, Hunt BJ, et al. Strategies for prevention and management of bleeding following pediatric cardiac surgery on cardiopulmonary bypass: a scoping review. Pediatr Crit Care Med 2018:19:40-7.

2 Cholette JM, Faraoni D, Goobie SM, et al. Patient blood management in pediatric cardiac surgery: a review. Anesth Analg 2018;127:1002-16.

3 Koster A, Faraoni D, Levy JH. Antifibrinolytic therapy for cardiac surgery: an update. Anesthesiology 2015;123:214-21.

4 Basta MN, Stricker PA, Taylor JA. A systematic review of the use of antifibrinolytic agents in pediatric surgery and implications for craniofacial use. Pediatr Surg Int 2012;28:1059-69.

5 Faraoni D, Willems A, Melot C, et al. Efficacy of tranexamic acid in paediatric cardiac surgery: a systematic review and meta-analysis. Eur J Cardiothorac Surg 2012;42:781-6.

6 Kozek-Langenecker SA, Ahmed AB, Afshari A, et al. Management of severe perioperative bleeding: guidelines from the European Society of Anaesthesiology: first update 2016. Eur J Anaesthesiol 2017;34:332-95.

7 Zhang Y, Zhang X, Wang Y, et al. Efficacy and safety of tranexamic acid in pediatric patients undergoing cardiac surgery: a single-center experience. Front Pediatr 2019;7.

8 Shimizu K, Toda Y, Iwasaki T, et al. Effect of tranexamic acid on blood loss in pediatric cardiac surgery: a randomized trial. J Anesth 2011;25:823-30.
9 Pasquali SK, Li JS, He X, et al. Comparative analysis of antifibrinolytic medications in pediatric heart surgery. $J$ Thorac Cardiovasc Surg 2012;143:550-7.

10 Myles PS, Smith JA, Forbes A, et al. Tranexamic acid in patients undergoing coronary-artery surgery. N Engl J Med 2017;376:136-48.

11 Lin Z, Xiaoyi Z. Tranexamic acid-associated seizures: a metaanalysis. Seizure 2016;36:70-3.

12 Takagi $\mathrm{H}$, Ando T, Umemoto T, et al. Seizures associated with tranexamic acid for cardiac surgery: a meta-analysis of randomized and non-randomized studies. J Cardiovasc Surg 2017;58:633-41.

13 Breuer T, Martin K, Wilhelm M, et al. The blood sparing effect and the safety of aprotinin compared to tranexamic acid in paediatric cardiac surgery. Eur J Cardiothorac Surg 2009;35:167-71.

14 Martin K, Breuer T, Gertler R, et al. Tranexamic acid versus $\varepsilon$-aminocaproic acid: efficacy and safety in paediatric cardiac surgery. Eur J Cardiothorac Surg 2011;39:892-7.

15 Maeda T, Sasabuchi $\mathrm{Y}$, Matsui $\mathrm{H}$, et al. Safety of tranexamic acid in pediatric cardiac surgery: a nationwide database study. $J$ Cardiothorac Vasc Anesth 2017;31:549-53.

16 Manji RA, Grocott HP, Leake J, et al. Seizures following cardiac surgery: the impact of tranexamic acid and other risk factors. Can J Anaesth 2012;59:6-13.

17 Gaynor JW, Jarvik GP, Gerdes M, et al. Postoperative electroencephalographic seizures are associated with deficits in executive function and social behaviors at 4 years of age following cardiac surgery in infancy. $J$ Thorac Cardiovasc Surg 2013;146:132-9.

18 Mehta RL, Kellum JA, Shah SV, et al. Acute kidney injury network: report of an initiative to improve outcomes in acute kidney injury. Crit Care 2007;11.

19 Giordano R, Palma G, Poli V, et al. Tranexamic acid therapy in pediatric cardiac surgery: a single-center study. Ann Thorac Surg 2012:94:1302-6.

20 Nishijima DK, Monuteaux MC, Faraoni D, et al. Tranexamic acid use in United States Children's Hospitals. J Emerg Med 2016;50:868-74.

21 Gertler R, Gruber M, Grassin-Delyle S, et al. Pharmacokinetics of tranexamic acid in neonates and infants undergoing cardiac surgery. Br J Clin Pharmacol 2017;83:1745-57.

22 Rozen L, Faraoni D, Sanchez Torres C, et al. Effective tranexamic acid concentration for $95 \%$ inhibition of tissue-type plasminogen activator induced hyperfibrinolysis in children with congenital heart disease: A prospective, controlled, in-vitro study. Eur J Anaesthesiol 2015;32:844-50.

23 Wesley MC, Pereira LM, Scharp LA, et al. Pharmacokinetics of tranexamic acid in neonates, infants, and children undergoing cardiac surgery with cardiopulmonary bypass. Anesthesiology 2015;122:746-58.

24 Faraoni D, Rahe C, Cybulski KA. Use of antifibrinolytics in pediatric cardiac surgery: where are we now? Paediatr Anaesth 2019;29:435-40.

25 Lin C-Y, Shuhaiber JH, Loyola H, et al. The safety and efficacy of antifibrinolytic therapy in neonatal cardiac surgery. PLoS One 2015;10:e0126514 